\title{
Casimir effect at nonzero temperature for wedges and cylinders
}

\author{
Simen Å. Ellingsen $\circledast$ and Iver Breviki \\ Department of Energy and Process Engineering, Norwegian University of Science and Technology, N-7491 Trondheim, Norway \\ Kimball A. Milton \\ Oklahoma Center for High Energy Physics and Homer L. Dodge Department of Physics and Astronomy, \\ The University of Oklahoma, Norman, OK 73019, USA \\ (Dated: October 25, 2018)

\begin{abstract}
We consider the Casimir-Helmholtz free energy at nonzero temperature $T$ for a circular cylinder and perfectly conducting wedge closed by a cylindrical arc, either perfectly conducting or isorefractive. The energy expression at nonzero temperature may be regularized to obtain a finite value, except for a singular corner term in the case of the wedge which is present also at zero temperature. Assuming the medium in the interior of the cylinder or wedge be nondispersive with refractive index $n$, the temperature dependence enters only through the non-dimensional parameter $2 \pi n a T, a$ being the radius of the cylinder or cylindrical arc. We show explicitly that the known zero temperature result is regained in the limit $a T \rightarrow 0$ and that previously derived high temperature asymptotics for the cylindrical shell are reproduced exactly.

PACS numbers: 42.50.Lc, 11.10.Wx, 11.10.Gh, 42.50.Pq
\end{abstract}

\section{INTRODUCTION}

The Casimir effect [1] is the name given to energies and forces due to field fluctuations in the presence of boundaries. Once a theoretical curiosity, the effect has gained enormous and still increasing attention since its first quantitative measurement a good decade ago [2] Reviews of recent progress include Refs. $3-5]$.

The first geometry, considered in Casimir's classic paper [1] was that of two perfectly conducting plates, generalized to arbitrary dispersive materials by Lifshitz [6]. The force between parallel plates of any purely dielectric material is attractive, and it was therefore surprising when it was shown by Boyer that the Casimir stress on a perfectly conducting spherical shell is repulsive [7].

While it was clear from Boyer's result that the Casimir effect has a strong geometry dependence, results for new geometries were slow in coming for a long time, and it was only in 1981 that DeRaad and Milton calculated the Casimir energy for a circularly cylindrical shell [8]. Since then a number of analytical efforts have added to the knowledge of Casimir effect in cylindrical cavities, both perfectly conducting $9-12$ and (magneto)dielectric 13 18]. Most treatments of the cylindrical geometry have dealt with the zero temperature situation, and only a few calculations have concerned finite temperature [19 21], and in these references only the high-temperature asymptotics were derived. No analytical expression valid for all temperatures exists for the cylindrical geometry to our knowledge.

A related geometry is the wedge. First considered with

\footnotetext{
*Electronic address: simen.a.ellingsen@ntnu.no

${ }^{\dagger}$ Electronic address: iver.h.brevik@ntnu.no

‡Electronic address: milton@nhn.ou.edu
}

respect to Casimir effect in the 1970s 22, 23], it has been the subject of several treatments later $24-28]$. The geometry is inviting in that it is analytically solvable and contains the geometries of parallel plates and a single semi-infinite plate as limiting cases. The geometry of a wedge intercut by a cylindrical shell was considered by Nesterenko and co-workers [29, 30] and energy densities in the same geometry were calculated by Saharian and co-workers [31 33]. We are not aware of any previous efforts to tackle the Casimir energy problem for a wedge at non-zero temperature.

We recently revisited the latter geometry to calculate the energy, at zero temperature, of a perfectly conducting wedge closed by a cylindrical boundary, either perfectly conducting or magnetodielectric [34]. We showed how that energy could be written on the form (subscript 0 indicates zero temperature)

$$
\mathcal{E}_{0}=\tilde{\mathcal{E}}_{0}(p)+\hat{\mathcal{E}}
$$

where $\tilde{\mathcal{E}}_{0}$ is a finite, regularizable energy closely analogous to that found for a cylinder [8, 10], whereas $\hat{\mathcal{E}}$ is a divergent term associated with the corners where the arc meets the wedge.

Here and in the following we will make frequent use of the symbol

$$
p=\pi / \alpha,
$$

where the physical range is $p \in\left[\frac{1}{2}, \infty\right)$, but which we will in general allow to take any real positive value. Throughout our calculations we set $c=\hbar=k_{\mathrm{B}}=1$. It was shown 34] that $\hat{\mathcal{E}}$ could be rendered finite provided the arc become transparent at high frequencies.

The calculations in [34] were extended to the first consideration of a wedge which is not perfectly conducting but instead assumed to be isorefractive (diaphanous), i.e., spatially uniform speed of light [35]. In that case 
the term $\hat{\mathcal{E}}$ is not present at all. The diaphanous wedge is analogous to the system of an annular region between two perfectly conducting cylinders, intercut by two semitransparent, radially directed interfaces [36, 37]. Notably, while the energy expressions for a perfectly conducting wedge or circularly cylindrical shell require some regularization scheme in order to give numerical meaning, the energy expression obtained for the diaphanous wedge is immediately finite. A review of the Casimir wedge problem and an early exposition of the issue we elaborate herein are found in Ref. [38].

Naturally, for the geometry of a perfectly conducting cylinder there is no divergent term $\hat{\mathcal{E}}$ since there are no sharp corners. It turns out (c.f. the discussion in Section III of Ref. [34]) that the Casimir energy of a perfectly conducting cylindrical shell is

$$
\tilde{\mathcal{E}}_{\text {cyl }}=2 \tilde{\mathcal{E}}(p=1)
$$

Thus, all of the calculations in the following sections, which are carried out for general $p$, are valid also for a cylindrical shell by letting $p \rightarrow 1$ and multiplying by an overall factor of 2 .

In the following we derive an analytical expression for the Casimir energy of a perfectly conducting wedge (modulo a singular term as encountered in the past) and a perfectly conducting cylindrical shell, valid for arbitrary opening angles and all temperatures. This extends the calculations for the perfectly conducting wedge presented in [34], and simultaneously those for a circularly cylindrical conducting shell [8, 10], to the case of finite temperature. We show how the energy expression, which for $T>0$ is the Helmholtz free energy, may be regularized by a scheme of Epstein-zeta functions to obtain a numerically useful expression. We show explicitly that the expression thus obtained reduces to the previously derived zero temperature limit, and that the two leading terms of the high temperature asymptotic expansion, derived by Bordag, Nesterenko and Pirozhenko [21], are reproduced exactly as a special case.

\section{CASIMIR-HELMHOLTZ FREE ENERGY OF WEDGE AND CYLINDER}

We take as our starting point the zero temperature energy derived for the geometry of a perfectly conducting wedge of opening angle $\alpha$ closed by a perfectly conducting cylindrical arc of radius $a$, derived in [34], shown on the left side of Fig. 1.

Henceforth we shall focus on the term $\tilde{\mathcal{E}}_{0}$, which may be written 34]

$$
\tilde{\mathcal{E}}_{0}=\sum_{m=0}^{\infty}{ }^{\prime} \mathcal{E}_{m 0}
$$
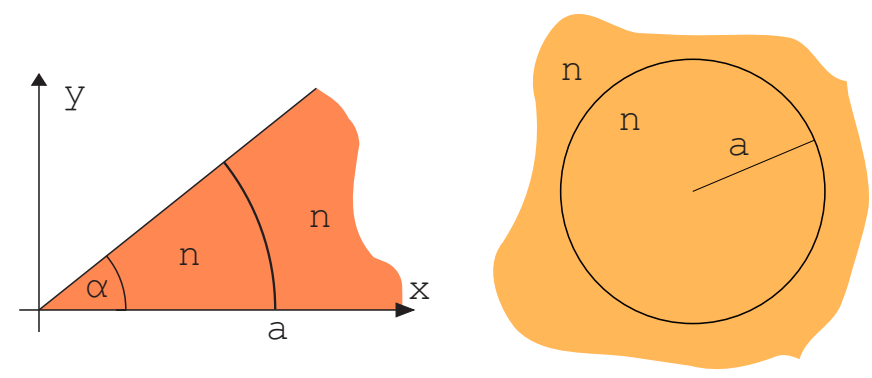

FIG. 1: The geometry considered: (left) a wedge of opening angle $\alpha$ closed by a cylindrical shell at radius $a$. The results are automatically applicable to a cylindrical shell of radius $a$ (right) when $\alpha=\pi$ [equation (1.3)].

with

$$
\begin{aligned}
\mathcal{E}_{m 0}= & -\frac{1}{8 \pi^{2}} \int_{-\infty}^{\infty} d k \int_{-\infty}^{\infty} d \zeta \zeta \\
& \times \frac{d}{d \zeta} \ln I_{m p}(x) I_{m p}^{\prime}(x) K_{m p}(x) K_{m p}^{\prime}(x)
\end{aligned}
$$

where we define the shorthand $x^{2}=a^{2}\left(k^{2}+n^{2} \zeta^{2}\right)$ where $n$ is the index of refraction of the medium inside the wedge. We assume $n$ to be constant with respect to $\zeta$ and uniform in space. Here $\omega=i \zeta$ is the reciprocal of imaginary (Euclidian) time. By means of partial integration with respect to $\zeta$, adding a trivial constant and noting that the integrand is symmetrical under $\zeta \rightarrow-\zeta$ and $k \rightarrow-k$, this may be written on the familiar form

$$
\mathcal{E}_{m 0}=\frac{1}{2 \pi^{2}} \int_{0}^{\infty} d k \int_{0}^{\infty} d \zeta \ln \left[1-x^{2} \lambda_{m p}^{2}(x)\right],
$$

wherein we use the shorthand

$$
\lambda_{\nu}(x)=\frac{d}{d x}\left[I_{\nu}(x) K_{\nu}(x)\right] .
$$

The Helmholtz free energy at $T>0$ is obtained from the 'trace-log' formula (2.3) by compactifying the Euclidean time axis as is well known. Technically this amounts to the transition

$$
\int_{0}^{\infty} d \zeta f(\zeta) \rightarrow 2 \pi T \sum_{j=0}^{\infty} f\left(\zeta_{j}\right),
$$

where $\zeta_{j}=2 \pi j T$ are the Matsubara frequencies. Changing the integration variable from axial momentum $k$ to $x$, the resulting expression for the finite part of the free energy may be written

$$
\tilde{\mathcal{E}}=\frac{T}{\pi a} \sum_{m=0}^{\infty} \sum_{j=0}^{\infty} e_{m, j},
$$

where

$$
e_{m, j}(\tau, p)=\int_{j \tau}^{\infty} \frac{d x x}{\sqrt{x^{2}-j^{2} \tau^{2}}} \ln \left[1-x^{2} \lambda_{m p}^{2}(x)\right],
$$


and where we have defined the dimensionless temperature

$$
\tau=2 \pi n a T
$$

Similarly to the case at zero temperature this simple expression is in need of regularization in order to give numerical meaning since it is formally divergent.

\section{REGULARIZATION OF THE FREE ENERGY EXPRESSION}

We here follow a scheme closely reminiscent of that of Milton and DeRaad [8], and particularly Milton, Nesterenko and Nesterenko [10] (cf. also Appendix A of Ref. [34]).

As follows from the uniform asymptotic expansion of modified cylindrical Bessel functions, e.g. $§ 9.7$ of Ref. [39], the logarithmic factor in the integrand of (2.7) has the asymptotic behavior

$$
\begin{aligned}
\ln \left[1-x^{2} \lambda_{m p}^{2}\right] & \sim-\frac{x^{4}}{4\left(m^{2} p^{2}+x^{2}\right)^{3}}, & m, x \rightarrow \infty \\
\ln \left[1-x^{2} \lambda_{0}^{2}\right] & \sim-\frac{x^{4}}{4\left(1+x^{2}\right)^{3}}, & x \rightarrow \infty
\end{aligned}
$$

To see how this behavior gives rise to a formal divergence, consider the case of large $m$ for which

$$
\begin{aligned}
e_{m, j} & \sim-\frac{1}{4} \int_{j \tau}^{\infty} \frac{d x x^{5}}{\sqrt{x^{2}-j^{2} \tau^{2}}\left(m^{2} p^{2}+x^{2}\right)^{3}} \\
& =-\frac{1}{4} \int_{0}^{\infty} \frac{d y\left(y^{4}+2 j^{2} \tau^{2} y^{2}+j^{4} \tau^{4}\right)}{\left(m^{2} p^{2}+j^{2} \tau^{2}+y^{2}\right)^{3}} \\
& =-\frac{3 \pi}{64 \varphi}-\frac{\pi j^{2} \tau^{2}}{32 \varphi^{3}}-\frac{3 \pi j^{4} \tau^{4}}{64 \varphi^{5}}
\end{aligned}
$$

where we substituted $y^{2}=x^{2}-j^{2} \tau^{2}$ and defined the shorthand

$$
\varphi=\sqrt{p^{2} m^{2}+\tau^{2} j^{2}}
$$

The three terms of $3.2 \mathrm{~b}$ correspond to the three terms of the integrand of (3.2a). All of the terms of (3.2b) clearly diverge when summed over $j$ and $m$.

The first step in regularization is to add and subtract the asymptotic behavior (3.1) in the form (3.2a)

$$
\tilde{\mathcal{E}}=\overline{\mathcal{E}}+\mathcal{E}_{\infty}
$$

where we define the energy with the leading asymptotic term subtracted,

$$
\begin{aligned}
\overline{\mathcal{E}}= & \frac{T}{\pi a} \sum_{m=0}^{\infty} \sum_{j=0}^{\infty} \tilde{e}_{m, j} \\
\tilde{e}_{m, j}= & \int_{j \tau}^{\infty} \frac{d x x}{\sqrt{x^{2}-j^{2} \tau^{2}}} \\
& \times\left\{\ln \left[1-x^{2} \lambda_{m p}^{2}(x)\right]+\frac{x^{4}}{4\left(m^{2} p^{2}+x^{2}\right)^{3}}\right\} \\
\tilde{e}_{0, j}= & \int_{j \tau}^{\infty} \frac{d x x}{\sqrt{x^{2}-j^{2} \tau^{2}}} \\
& \times\left\{\ln \left[1-x^{2} \lambda_{0}^{2}(x)\right]+\frac{x^{4}}{4\left(1+x^{2}\right)^{3}}\right\}
\end{aligned}
$$

and the additional, non-regularized energy

$$
\mathcal{E}_{\infty}=-\frac{T}{4 \pi a} \sum_{m=0}^{\infty} \sum_{j=0}^{\prime} \int_{0}^{\infty} \frac{d y\left(y^{4}+2 j^{2} \tau^{2} y^{2}+j^{4} \tau^{4}\right)}{\left(\tilde{\varphi}^{2}+y^{2}\right)^{3}}
$$

wherein

$$
\tilde{\varphi}=\left\{\begin{array}{cc}
\sqrt{1+\tau^{2} j^{2}}, & m=0 \\
\sqrt{p^{2} m^{2}+\tau^{2} j^{2}}, & m \geq 1
\end{array} .\right.
$$

To regularize $\mathcal{E}_{\infty}$ we introduce the small quantity $s$ and write

$$
\begin{aligned}
\mathcal{E}_{\infty}= & -\frac{T}{4 \pi a} \lim _{s \rightarrow 0^{+}} \sum_{m=0}^{\infty} \sum_{j=0}^{\infty} \int_{0}^{\infty} \frac{d y y^{-s}}{\left(\tilde{\varphi}^{2}+y^{2}\right)^{3}} \\
& \times\left(y^{4}+2 j^{2} \tau^{2} y^{2}+j^{4} \tau^{4}\right) \\
\sim & -\frac{T}{64 a} \lim _{s \rightarrow 0^{+}} \sum_{m=0}^{\infty} \sum_{j=0}^{\infty}\left\{\frac{3}{\tilde{\varphi}^{1+s}}\right. \\
& \left.+\frac{2 j^{2} \tau^{2}}{\tilde{\varphi}^{3+s}}+\frac{3 j^{4} \tau^{4}}{\tilde{\varphi}^{5+s}}\right\},
\end{aligned}
$$

where as in Eq. (3.2b) we have used the evaluation,

$$
\int_{0}^{\infty} \frac{d y y^{4-s}}{\left(\tilde{\varphi}^{2}+y^{2}\right)^{3}}=\frac{\pi(1-s)(3-s)}{16 \tilde{\varphi}^{1+s}} \sec \frac{\pi s}{2},
$$

which is valid for $-1<s<5$, so it may be used for $s$ near 0 , near 2 , or near 4 . We use the relations $\left[\partial_{\tau}=\partial / \partial \tau\right]$

$$
\begin{aligned}
& \partial_{\tau} \frac{1}{\tilde{\varphi}^{q}}=-\frac{q \tau j^{2}}{\tilde{\varphi}^{q+2}} \\
& \partial_{\tau}^{2} \frac{1}{\tilde{\varphi}^{q}}=-\frac{q j^{2}}{\tilde{\varphi}^{q+2}}+\frac{q(q+2) \tau^{2} j^{4}}{\tilde{\varphi}^{q+4}}
\end{aligned}
$$

to write

$$
\begin{aligned}
\mathcal{E}_{\infty}= & -\frac{T}{64 a}\left(3-3 \tau \partial_{\tau}+\tau^{2} \partial_{\tau}^{2}\right) \\
& \times \lim _{s \rightarrow 0^{+}} \sum_{m=0}^{\infty} \sum_{j=0}^{\infty} \frac{1}{\tilde{\varphi}^{1+s}} .
\end{aligned}
$$


The sum in (3.11) can be regularized by analytical continuation. We will write it in the following form, using symmetry properties with respect to $m \leftrightarrow-m$ and $j \leftrightarrow-j:$

$$
\begin{aligned}
\lim _{s \rightarrow 0^{+}} \sum_{m=0}^{\infty} \sum_{j=0}^{\infty} \frac{1}{\tilde{\varphi}^{1+s}} & =\frac{1}{4} \lim _{s \rightarrow 0^{+}} \sum_{m, j \in \mathbb{Z}} \frac{1}{\tilde{\varphi}^{1+s}} \\
& =\frac{1}{4}+\frac{1}{4} \mathcal{S}(\tau, p)+\frac{1}{2} \mathcal{K}(\tau) .
\end{aligned}
$$

Here we have defined

$$
\mathcal{S}(\tau, p)=\lim _{s \rightarrow 0^{+}} \sum_{m, j \in \mathbb{Z}}^{\prime \prime} \frac{1}{\varphi^{1+s}}=\mathcal{S}(p, \tau),
$$

wherein the double prime on the summation mark means that the term $m=j=0$ is explicitly excluded, and

$$
\begin{aligned}
\mathcal{K}(\tau) & =\lim _{s \rightarrow 0^{+}} \sum_{j=1}^{\infty}\left[\frac{1}{\tilde{\varphi}^{1+s}}-\frac{1}{\varphi^{1+s}}\right]_{m=0} \\
& =\sum_{j=1}^{\infty} \frac{j \tau-\sqrt{1+j^{2} \tau^{2}}}{j \tau \sqrt{1+j^{2} \tau^{2}}} .
\end{aligned}
$$

Clearly, $\mathcal{K}(\tau)$ is finite for all $\tau>0$.

The function $\mathcal{S}(\tau, p)$ may be regularized by use of the Chowla-Selberg formula [see e.g. Eq. (4.33) of Ref. [40]]

$$
\begin{aligned}
\sum_{m, j \in \mathbb{Z}}^{\prime \prime} & \left(a m^{2}+b m j+c j^{2}\right)^{-q}=2 \zeta(2 q) a^{-q} \\
& +\frac{2^{2 q} \sqrt{\pi} a^{q-1} \Gamma\left(q-\frac{1}{2}\right) \zeta(2 q-1)}{\Gamma(q) \Delta^{q-\frac{1}{2}}} \\
& +\frac{2^{q+\frac{5}{2}} \pi^{q}}{\Gamma(q) \Delta^{\frac{1}{2}\left(q-\frac{1}{2}\right)} \sqrt{a}} \sum_{l=1}^{\infty} l^{q-\frac{1}{2}} \sigma_{1-2 q}(l) \\
& \times \cos (l \pi b / a) K_{q-\frac{1}{2}}(\pi l \sqrt{\Delta} / a),
\end{aligned}
$$

where

$$
\begin{aligned}
\Delta & =4 a c-b^{2}, \\
\sigma_{w}(l) & =\sum_{\nu \mid l} \nu^{w},
\end{aligned}
$$

where $\nu$ are summed over the divisors of $l$ and it is assumed that $\Delta>0 . K$ is again the modified Bessel function of the second kind. The apparent pole as $q \rightarrow \frac{1}{2}$ now vanishes due to a cancellation between the first two terms of (3.15), and we find that letting $q=\frac{1}{2}+\frac{s}{2}$ and taking the limit $s \rightarrow 0^{+}$(here $a=p^{2}, b=0, c=\tau^{2}$ )

$$
\mathcal{S}(\tau, p)=\frac{2}{p}\left(\gamma-\ln \frac{4 \pi p}{\tau}\right)+\frac{8}{p} \sum_{l=1}^{\infty} \sigma_{0}(l) K_{0}(2 \pi l \tau / p)
$$

where $\gamma=0.577216 \ldots$ is Euler's constant. Now $\sigma_{0}(l)$ is simply the number of positive divisors of $l, \sigma_{0}(1)=$ $1, \sigma_{0}(2)=\sigma_{0}(3)=2, \sigma_{0}(4)=3$ etc. Note that Eq. (3.18)

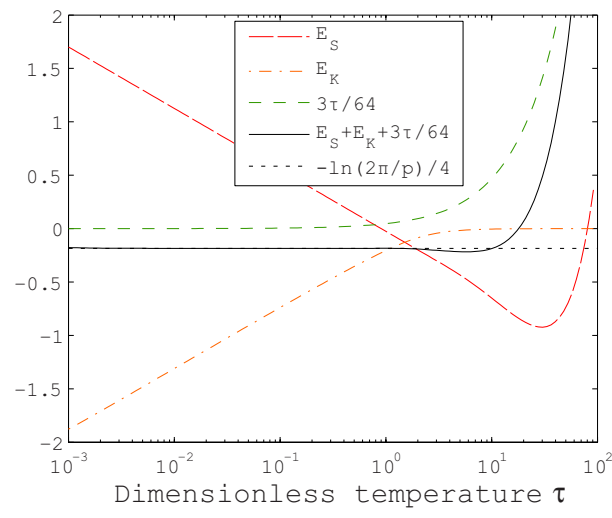

FIG. 2: The additional terms of the regularized energy in Eq. (3.20) which are subtracted from the double sum there in the case $p=3$. Shown also is the sum of the three additional terms and their low-temperature asymptotic value from Eq. (4.11.

is valid for all $\tau$; although it appears most convenient for large $\tau$, it is, by the symmetry property seen in Eq. (3.13), equally useful for small $\tau$.

We finally write down the final, regularized energy of the wedge (and, simultaneously, cylinder) at finite $T$, using the convention used in Ref. 34]

$$
\tilde{\mathcal{E}}(\tau, p, a)=\frac{1}{8 \pi n a^{2}} e(\tau, p),
$$

in terms of

$$
\begin{aligned}
& e(\tau, p)=\frac{4 \tau}{\pi} \sum_{m=0}^{\infty} \sum_{j=0}^{\infty} \tilde{e}_{m, j}(\tau, p) \\
& -\frac{\tau}{64}\left(3-3 \tau \partial_{\tau}+\tau^{2} \partial_{\tau}^{2}\right)[1+2 \mathcal{K}(\tau)+\mathcal{S}(\tau, p)] .
\end{aligned}
$$

with $\tilde{e}_{m, j}, \mathcal{K}$, and $\mathcal{S}$ given in Eqs. (3.5), (3.14) and (3.18), respectively. The differentiations with respect to $\tau$ are now straightforward, should the full expanded expression be desirable.

In Fig. 2 we plot the three additional terms in the second line of Eq. (3.20) where we have defined the shorthand

$$
\begin{aligned}
\hat{\mathcal{T}} & =\left(3-3 \tau \partial_{\tau}+\tau^{2} \partial_{\tau}^{2}\right) \\
E_{S}(\tau, p) & =\frac{\tau}{64} \hat{\mathcal{T}} \mathcal{S}(\tau, p) ; \quad E_{K}(\tau, p)=\frac{\tau}{32} \hat{\mathcal{T}} \mathcal{K}(\tau) .
\end{aligned}
$$

Figure 3 shows a numerical calculation of $e(\tau, p=3)$ as a function of $\tau$ along with its high and low $\tau$ asymptotes (see derivations in the following sections). The calculation was performed by "brute force" by truncating the sums after a number of terms, and has somewhat limited accuracy due to the large number of terms in the $j$ sum in Eq. (3.20) required for small $\tau$, scaling as $\tau^{-1}$. 


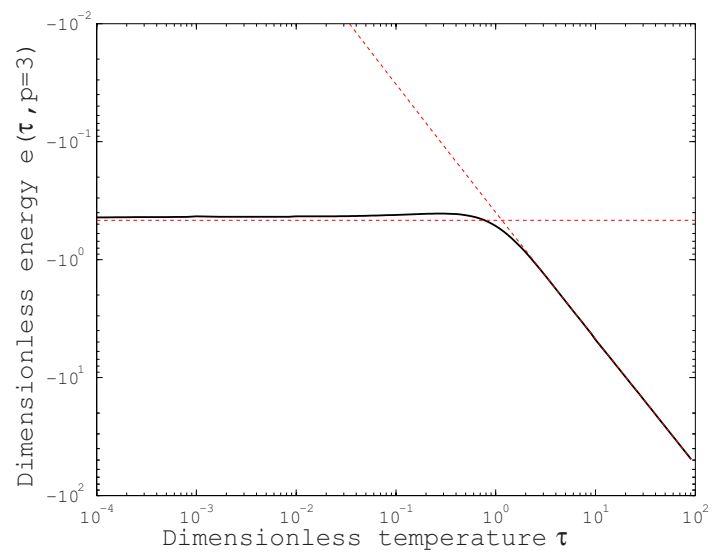

FIG. 3: Dimensionless energy $e(\tau, p)$ for the case $p=3$, i.e. opening angle $\alpha=\pi / 3$ approximated by a "brute force" calculation truncating the sums. The zero-temperature limit and high- $\tau$ asymptote are shown as dashed lines.

\section{REGAINING THE LIMIT OF ZERO TEMPERATURE}

Comparing Eq. (3.20) with the zero temperature result derived in Ref. 34], and previously known for the cylindrical shell [8, 10], it is not obvious that our expression simplifies to the zero temperature result as $\tau \rightarrow 0$. In this section we show that upon careful examination the correct limit is in fact obtained.

Let us write down the zero temperature result $\tilde{\mathcal{E}}_{0}$ for general $p$ in its regularized form suitable for comparison ${ }^{1}$ (c.f. Ref. 34], Eq. (4.14)):

$$
\begin{aligned}
& \tilde{\mathcal{E}}_{0}=\frac{1}{2} \overline{\mathcal{E}}_{0}+\sum_{m=1}^{\infty} \overline{\mathcal{E}}_{m}+\frac{1}{32 \pi n a^{2}} \ln (2 \pi / p) \\
& \overline{\mathcal{E}}_{0}=\frac{1}{4 \pi n a^{2}} \int_{0}^{\infty} d x x\left[\ln \left(1-x^{2} \lambda_{0}^{2}\right)+\frac{x^{4}}{4\left(1+x^{2}\right)^{3}}\right]
\end{aligned}
$$

$\overline{\mathcal{E}}_{m 0}=\frac{1}{4 \pi n a^{2}} \int_{0}^{\infty} d x x\left[\ln \left(1-x^{2} \lambda_{m p}^{2}\right)+\frac{x^{4}}{4\left(m^{2} p^{2}+x^{2}\right)^{3}}\right]$.

The finite temperature quantity $\overline{\mathcal{E}}$ of Eq. (3.5) is analytic as $\tau \rightarrow 0$ and inverse application of the transition (2.5) simply gives us

$$
\overline{\mathcal{E}} \stackrel{\tau \rightarrow 0}{\longrightarrow} \frac{1}{2} \overline{\mathcal{E}}_{0}+\sum_{m=1}^{\infty} \overline{\mathcal{E}}_{m}
$$

What remains is essentially to determine the low $\tau$ behavior of $\mathcal{K}(\tau)$ and $\mathcal{S}(\tau, a)$ to check that the last term of Eq. (4.1a) may be regained.

\footnotetext{
${ }^{1}$ These definitions of $\overline{\mathcal{E}}_{0}$ and $\overline{\mathcal{E}}_{m 0}$ differ from those of Ref. [34] by a prefactor $n$.
}

To study the behavior of $\mathcal{S}$ it is convenient to employ the symmetry relation $\mathcal{S}(\tau, p)=\mathcal{S}(p, \tau)$ which gives

$$
\mathcal{S}(\tau, p)=\frac{2}{\tau}\left(\gamma-\ln \frac{4 \pi \tau}{p}\right)+\frac{8}{\tau} \sum_{l=1}^{\infty} \sigma_{0}(l) K_{0}(2 \pi l p / \tau) .
$$

For large arguments $K_{0}(x) \propto \exp (-x)$, so the sum over $l$ is exponentially small as $\tau \rightarrow 0$. This immediately gives the asymptotic behavior:

$$
\mathcal{S}(\tau, p) \sim \frac{2}{\tau}[\gamma-\ln (2 \pi / p)-\ln 2-\ln \tau], \tau \rightarrow 0
$$

Next we turn to $\mathcal{K}(\tau)$. Using the Euler-Maclaurin formula (e.g. Ref. 39], p.806) we have

$$
\begin{aligned}
\mathcal{K}(\tau)= & \sum_{l=0}^{\infty} \varkappa(\tau+l \tau)=\frac{1}{\tau} \int_{\tau}^{\infty} d t \varkappa(t) \\
& +\frac{1}{2} \varkappa(\tau)-\sum_{l=1}^{\infty} \frac{\tau^{2 l-1} B_{2 l}}{(2 l) !} \varkappa^{(2 l-1)}(\tau)
\end{aligned}
$$

with

$$
\varkappa(t)=\frac{t-\sqrt{1+t^{2}}}{t \sqrt{1+t^{2}}}
$$

The integral has the solution

$$
\begin{aligned}
\int_{\tau}^{\infty} d t \varkappa(t) & =\ln \frac{2 \tau}{\tau+\sqrt{1+\tau^{2}}} \\
& =\ln \tau+\ln 2-\tau+\frac{1}{6} \tau^{3}+\ldots
\end{aligned}
$$

as $\tau \rightarrow 0$. Moreover, $\varkappa(\tau)=-1 / \tau+1-\frac{1}{2} \tau^{2}+.$. , and upon inspection we recognize that

$$
\tau^{2 l-1} \varkappa^{(2 l-1)}(\tau)=\frac{(2 l-1) !}{\tau}+(-1)^{l}\left[\frac{(2 l) !}{2^{l} l !}\right]^{2} \tau^{2 l}+\ldots
$$

To leading order in $\tau$, thus, the sum in Eq. (4.5) reads $\frac{1}{\tau} \sum_{l=1}^{\infty} B_{2 l} / 2 l$. As is typically the case for series expansions close to non-analytical points, the series is formally divergent. It can, however, be regularized by means of Borel summation [41]. For a highly similar problem and details on how to approach it, see [42]. We show in Appendix A that the Borel regularized sum evaluates to

$$
\sum_{l=1}^{\infty} \frac{B_{2 l}}{2 l}=\gamma-\frac{1}{2}
$$

Thus we have found the low- $\tau$ expansion of $\mathcal{K}(\tau)$ :

$$
\mathcal{K}(\tau) \sim \frac{1}{\tau}(\ln \tau+\ln 2-\gamma)-\frac{1}{2}+\ldots, \quad \tau \rightarrow 0
$$

Further terms cancel at least to order $\tau^{2}$, the leading order correction being at least of order $\tau^{4}$. 
Combining (4.4) and (4.10) we find, to leading order in $\tau$, the expression in square brackets in (3.20):

$$
[1+2 \mathcal{K}+\mathcal{S}] \sim-\frac{2}{\tau} \ln (2 \pi / p)+\mathcal{O}\left(\tau^{4}\right), \quad \tau \rightarrow 0
$$

and using

$$
\left(3-3 \tau \partial_{\tau}+\tau^{2} \partial_{\tau}^{2}\right) \frac{1}{\tau}=\frac{8}{\tau}
$$

we regain exactly the zero temperature result (4.1). As illustrated in Fig. 3 this limit is reached very rapidly as $\tau \rightarrow 0$. While we have ascertained in the above that the correction term in (4.11) is at least of order $\tau^{4}$, there is reason to suspect that the behavior is in fact exponential, as is the case for $\mathcal{S}$ as seen from Eq. (4.3).

\section{HIGH- $\tau$ ASYMPTOTICS: AGREEMENT WITH PREVIOUS RESULTS FOR CYLINDRICAL SHELL}

We will finally determine the asymptotic behavior in the limit $\tau \gg 1$. Here the contribution from $\overline{\mathcal{E}}$ is given by the zeroth Matsubara term only. Consider the reduced energy $e(\tau, p)$ of Eq. (3.20) in which

$$
\frac{4 \tau}{\pi} \sum_{m=0}^{\infty} \sum_{j=0}^{\infty} \tilde{e}_{m, j}(\tau, p) \sim \frac{2 \tau}{\pi} C(p), \quad \tau \rightarrow \infty
$$

with

$$
\begin{gathered}
C(p)=\frac{1}{2} \int_{0}^{\infty} d x\left[\ln \left(1-x^{2} \lambda_{0}^{2}\right)+\frac{x^{4}}{4\left(1+x^{2}\right)^{3}}\right] \\
+\sum_{m=1}^{\infty} \int_{0}^{\infty} d x\left[\ln \left(1-x^{2} \lambda_{m p}^{2}\right)+\frac{x^{4}}{4\left(m^{2} p^{2}+x^{2}\right)^{3}}\right] .
\end{gathered}
$$

Some numerical values are

$$
\begin{aligned}
& C(1)=-0.75814 ; \\
& C(2)=-0.76558 ; \\
& C(3)=-0.76645 .
\end{aligned}
$$

These values were obtained with Mathematica, including 100 terms in the sum while checking convergence.

The high- $\tau$ behavior of $\mathcal{S}$ is given immediately by Eq. (3.18):

$$
\mathcal{S}(\tau, p) \sim \frac{2}{p}(\gamma-\ln 4 \pi p+\ln \tau), \quad \tau \rightarrow \infty
$$

where the correction term is exponential, wherewith

$$
\tau \partial_{\tau} \mathcal{S} \sim \frac{2}{p} ; \quad \tau^{2} \partial_{\tau}^{2} \mathcal{S} \sim-\frac{2}{p} .
$$

To study the behavior of $\mathcal{K}(\tau)$ and its derivatives it is useful to define $\beta=1 / \tau$ and write $\mathcal{K}(\tau)=\beta \mathcal{H}(\beta)$ with

$$
\mathcal{H}(\beta)=\sum_{j=1}^{\infty} \frac{j-\sqrt{j^{2}+\beta^{2}}}{j \sqrt{j^{2}+\beta^{2}}} .
$$

With a litte calculation one ascertains that

$$
\tau\left(3-3 \tau \partial_{\tau}+\tau^{2} \partial_{\tau}^{2}\right) \mathcal{K}(\tau)=\left(8+7 \beta \partial_{\beta}+\beta^{2} \partial_{\beta}^{2}\right) \mathcal{H}(\beta) .
$$

When $\beta \rightarrow 0$ it is simple to see from

$$
\begin{aligned}
\mathcal{H}^{\prime}(\beta) & =-\beta \sum_{j=1}^{\infty}\left(j^{2}+\beta^{2}\right)^{-\frac{3}{2}} \\
\mathcal{H}^{\prime \prime}(\beta) & =\sum_{j=1}^{\infty} \frac{2 \beta^{2}-j^{2}}{\left(j^{2}+\beta^{2}\right)^{\frac{5}{2}}}
\end{aligned}
$$

that

$$
\mathcal{H}(\beta) \sim \frac{1}{2} \beta \mathcal{H}^{\prime}(\beta) \sim \frac{1}{2} \beta^{2} \mathcal{H}^{\prime \prime}(\beta) \sim-\frac{1}{2} \beta^{2} \zeta(3), \quad \beta \rightarrow 0 .
$$

Hence we can safely ignore the term involving $\mathcal{K}$ at high $\tau$.

Combining this, the high- $\tau$ behavior of $e(\tau, p)$ is

$$
\begin{aligned}
e(\tau, p) \sim \tau & {\left[\frac{2 C(p)}{\pi}-\frac{3 p+6(\gamma-\ln 4 \pi p)-8}{64 p}\right.} \\
& \left.-\frac{3}{32 p} \ln \tau\right] .
\end{aligned}
$$

The high temperature asymptotics of perfectly conducting spherical and cylindrical shells with vacuum inside and outside were calculated by Bordag, Nesterenko and Pirozhenko [20, 21] using the method of heat kernel coefficients. They, like us, found that the two leading order terms were of order $T$ and $T \ln T$ as $T \rightarrow \infty$. The latter of these terms had been worked out some time previously by Balian and Duplantier [19], who also found an approximate (though not very accurate) value for the former.

The result of the calculations reported in [21] was, in our notation

$$
\begin{aligned}
\tilde{\mathcal{E}}_{\text {cyl }} & \sim-0.22924 \frac{T}{a}-\frac{3 T}{64 a} \ln \frac{a T}{2}+\mathcal{O}\left(T^{-1}\right) \\
& =-\frac{\tau}{8 \pi a^{2}}\left[0.44237+\frac{3}{16} \ln \tau+\mathcal{O}\left(\tau^{-2}\right)\right] .
\end{aligned}
$$

As previously mentioned, $\tilde{\mathcal{E}}_{\text {cyl }}=2 \tilde{\mathcal{E}}_{p=1}$. With the expansion (5.8) we find, using (5.3a),

$$
\begin{aligned}
2 \tilde{\mathcal{E}}_{p=1} \sim & -\frac{\tau}{8 \pi a^{2}}\left[\frac{6 \gamma-5-6 \ln 4 \pi}{32}-\frac{4}{\pi} C(1)\right. \\
& \left.+\frac{3}{16} \ln \tau+\ldots\right] \\
= & -\frac{\tau}{8 \pi a^{2}}\left[0.44270+\frac{3}{16} \ln \tau+\ldots\right] .
\end{aligned}
$$

The slight numerical difference we believe to be due to the approximate numerical method used in 21]. We show analytically in Appendix B that the correspondence is in fact exact. 

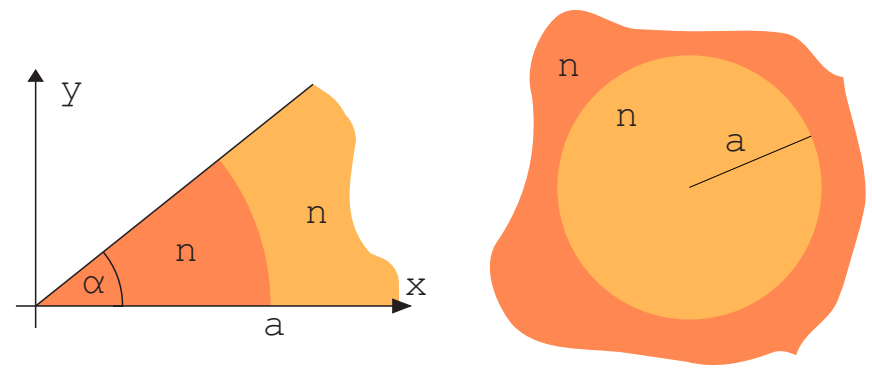

FIG. 4: Same geometry as in figure 1 but now with diaphanous instead of perfectly conducting arc, i.e., so that $n^{2}=\varepsilon \mu$ is the same both sides of the interface. We still assume nondispersive media.

\section{WEDGE WITH DIAPHANOUS ARC}

The above results can easily be extended to the case where the perfectly conducting arc is replaced by a diaphanous arc, that is, a magnetodielectric interface so that the product $n^{2}=\varepsilon \mu$ is the same for radii both smaller than and greater than $a$. This geometry was considered at zero temperature in Ref. 34]. The electromagnetic boundary conditions at the arc separate in a simple way in this case and the dependence on material properties enter only through the reflection coefficient

$$
\xi=\frac{\varepsilon_{2}-\varepsilon_{1}}{\varepsilon_{2}+\varepsilon_{1}}=-\frac{\mu_{2}-\mu_{1}}{\mu_{2}+\mu_{1}} .
$$

The change in geometry leaves the energy expression (2.3) unaltered but for the simple replacement

$$
\ln \left[1-x^{2} \lambda_{m p}^{2}(x)\right] \rightarrow \ln \left[1-\xi^{2} x^{2} \lambda_{m p}^{2}(x)\right]
$$

This merely introduces a prefactor $\xi^{2}$ in all correction terms, and we can write down the result for the diaphanous wedge, and simultaneously cylinder (by letting $p=1$ and multiplying by 2 as discussed above), as:

$$
\begin{aligned}
\tilde{\mathcal{E}}_{\xi}(\tau, p, a)= & \frac{1}{8 \pi n a^{2}} e_{\xi}(\tau, p) \\
e_{\xi}(\tau, p)= & \frac{4 \tau}{\pi} \sum_{m=0}^{\infty} \sum_{j=0}^{\infty} \tilde{e}_{m, j}(\tau, p, \xi)-\frac{\tau \xi^{2}}{64}\left(3-3 \tau \partial_{\tau}\right. \\
& \left.+\tau^{2} \partial_{\tau}^{2}\right)[1+2 \mathcal{K}(\tau)+\mathcal{S}(\tau, p)]
\end{aligned}
$$

wherein

$$
\begin{aligned}
\tilde{e}_{m, j}(\tau, p, \xi)= & \int_{j \tau}^{\infty} \frac{d x x}{\sqrt{x^{2}-j^{2} \tau^{2}}}\left\{\ln \left[1-\xi^{2} x^{2} \lambda_{m p}^{2}(x)\right]\right. \\
& \left.+\frac{\xi^{2} x^{4}}{4\left(m^{2} p^{2}+x^{2}\right)^{3}}\right\} ; \\
\tilde{e}_{0, j}(\tau, \xi)= & \int_{j \tau}^{\infty} \frac{d x x}{\sqrt{x^{2}-j^{2} \tau^{2}}}\left\{\ln \left[1-\xi^{2} x^{2} \lambda_{0}^{2}(x)\right]\right. \\
& \left.+\frac{\xi^{2} x^{4}}{4\left(1+x^{2}\right)^{3}}\right\} .
\end{aligned}
$$

Since $\xi$ enters the correction terms from renormalization only through the prefactor, generalization of the weak-coupling expansions (to leading order in $\xi^{2}$ ) considered in [34] to nonzero $\tau$ is trivial.

\section{CONCLUDING REMARKS}

We have given for the first time results for the temperature dependence of the Casimir energy for a wedge, closed by a circular arc, all boundaries being perfectly conducting. This includes, as a special case, the perfectly conducting cylindrical shell case. (Except for that case, there is a divergent term, due to the corner where the circular arc meets the wedge boundaries, which we here simply omit.) The low temperature result agrees with the zero-temperature result found previously, except for what is probably an exponentially small correction, while the high temperature result agrees with that of Bordag, Nesterenko, and Pirozhenko for the case of a cylinder [21].

\section{Acknowledgments}

The work of KAM was supported in part by grants from the US National Science Foundation and the US Department of Energy.

\section{Appendix A: Evaluation of Eq. (4.9) by Borel summation}

To evaluate a (possibly divergent) series $Z=\sum_{l=1}^{\infty} a_{l}$ by Borel summation [41] we define the function

$$
\phi(x)=\sum_{l=1}^{\infty} \frac{a_{l}}{l !} x^{l}
$$

If $\phi(x)$ is finite for sufficiently small $x$, we define the Borel transform as

$$
\mathcal{B}(x)=\int_{0}^{\infty} d t e^{-t} \phi(x t),
$$

from which the Borel regularized value of the sum $Z$ is $Z=\mathcal{B}(1)$. We consider the sum

$$
\sum_{l=1}^{\infty} \frac{B_{2 l}}{2 l}=\frac{1}{2}+\sum_{l=1}^{\infty} \frac{B_{l}}{l}
$$

since $B_{1}=-1 / 2$ and $B_{3}=B_{5}=B_{7}=\ldots=0$. The Borel transform of the latter sum is thus

$$
\mathcal{B}(x)=\int_{0}^{\infty} d t e^{-t} \sum_{l=1}^{\infty} \frac{B_{l}}{l \cdot l !}(x t)^{l} .
$$


The generating function of the Bernoulli numbers is

$$
\sum_{l=1}^{\infty} \frac{B_{l}}{l !} y^{l}=\frac{y}{e^{y}-1}-1
$$

to evaluate

$$
\begin{aligned}
\frac{d \mathcal{B}}{d x}(x) & =\frac{1}{x} \int_{0}^{\infty} d t e^{-t} \sum_{l=1}^{\infty} \frac{B_{l}}{l !}(x t)^{l}=\int_{0}^{\infty} \frac{d t t e^{-t}}{e^{x t}-1}-\frac{1}{x} \\
& =x^{-2} \int_{0}^{\infty} \frac{d u u e^{-u \frac{x+1}{x}}}{1-e^{-u}}-\frac{1}{x}=x^{-2} \psi^{(1)}\left(\frac{x+1}{x}\right)-\frac{1}{x}
\end{aligned}
$$

where $\psi^{(n)}(x)$ is the polygamma function, whose integral representation was recognized (Ref. [39] Eq. 6.4.1) by making the substitution $u=x t$. Thus we evaluate the integral to

$$
\begin{aligned}
\mathcal{B}(x)+\ln x & =\int^{x} \frac{d y}{y^{2}} \psi^{(1)}\left(\frac{y+1}{y}\right)=-\int^{\frac{x+1}{x}} d v \psi^{(1)}(v) \\
& =-\psi\left(\frac{x+1}{x}\right)+\text { constant, }
\end{aligned}
$$

where from the requirement that $\mathcal{B}(0)=0$ we see that the integration constant is zero. Thus we find the Borel value of the sum (A3) to be

$$
\sum_{l=1}^{\infty} \frac{B_{2 l}}{2 l}=\frac{1}{2}+\mathcal{B}(1)=\gamma-\frac{1}{2}
$$

noting that $\psi(2)=1-\gamma$.

\section{Appendix B: Correspondence with high- $T$ asymptotics for the cylinder in vacuum}

The heat kernel expansion for high temperatures calculated in Ref. [21] for the cylindrical shell in vacuum begins

$$
\begin{aligned}
\tilde{\mathcal{E}}_{\mathrm{cyl}} & \sim-\frac{T}{2} \zeta^{\prime}(0)-\frac{a_{3 / 2}}{(4 \pi)^{3 / 2}} T \ln T+\ldots \\
& =-\frac{\tau}{8 \pi a^{2}}\left[2 a \zeta^{\prime}(0)-\frac{3}{16} \ln 2 \pi a+\frac{3}{16} \ln \tau+\ldots\right]
\end{aligned}
$$

where the 'zeta determinant' $\zeta^{\prime}(0)$ is a constant defined in Ref. [21] and we have inserted their value [20, 21]

$$
\frac{a_{3 / 2}}{(4 \pi)^{3 / 2}}=\frac{3}{64 a}
$$

The term proportional to $\tau \ln \tau$ is obviously identical to our expression in Eq. (5.10). We consider only the term linear in $\tau$. Comparison with (5.10) gives, with minimal manipulation, that the asymptotes correspond exactly according to $\tilde{\mathcal{E}}_{\text {cyl }}=2 \tilde{\mathcal{E}}_{p=1}$, provided

$$
\pi a \zeta^{\prime}(0)+2 C(1)=\frac{\pi}{64}\left[6 \gamma-5+6 \ln \frac{a}{2}\right] .
$$

In Appendix B of [21] we find the following expression

$$
\begin{aligned}
& \pi a \zeta^{\prime}(0)=\int_{0}^{\infty} d y y \frac{d}{d y} \ln \left[1-y^{2} \lambda_{0}^{2}(y)\right] \\
& +2 \sum_{m=1}^{\infty} m \int_{0}^{\infty} d y y \frac{d}{d y}\left\{\ln \left[1-m^{2} y^{2} \lambda_{m}^{2}(m y)+\frac{y^{4} t^{6}}{4 m^{2}}\right]\right\} \\
& +\frac{\pi}{32}\left(3 \gamma-4+3 \ln \frac{a}{2}\right)
\end{aligned}
$$

with $t=1 / \sqrt{1+y^{2}}$. Let us call the two integrals in (B4) $X_{0}$ and $X_{m}$, where the latter is the integral inside the sum. After a partial integration and, in the case of $X_{m}$, a substitution $y m=x$, these can be written on the familiar form

$$
\begin{aligned}
X_{0}= & \left.-\int_{0}^{\infty} d x \ln \left[1-x^{2} \lambda_{0}^{2}(x)\right]\right] \\
X_{m}= & -\frac{1}{m} \int_{0}^{\infty} d x\left\{\ln \left[1-x^{2} \lambda_{m}^{2}(x)\right]\right. \\
& \left.\left.+\frac{z^{4}}{4\left(m^{2}+x^{2}\right)^{3}}\right]\right\} .
\end{aligned}
$$

Comparing with (5.2) we see that

$$
\begin{aligned}
\pi a \zeta^{\prime}(0)+2 C(1) & =\int_{0}^{\infty} \frac{d x x^{4}}{4\left(1+x^{2}\right)^{3}}+\frac{\pi}{32}\left(3 \gamma-4+3 \ln \frac{a}{2}\right) \\
& =\frac{\pi}{64}\left[6 \gamma-5+6 \ln \frac{a}{2}\right]
\end{aligned}
$$

since $\frac{1}{4} \int_{0}^{\infty} d x x^{4} /\left(1+x^{2}\right)^{3}=3 \pi / 64$. We have thus shown the correspondence analytically.
[1] H. B. G. Casimir, Proc. Kon. Ned. Akad. Wetensch. 51, 793 (1948).

[2] S. K. Lamoreaux, Phys. Rev. Lett. 78, 5 (1997).

[3] M. Bordag, G. L. Klimchitskaya, U. Mohideen, and V. M. Mostepanenko, Advances in the Casimir Effect (Oxford: Oxford University Press, 2009)

[4] K. A. Milton, J. Phys. A: Math. Gen. 37, R209 (2004).

[5] K. A. Milton The Casimir Effect: Physical Manifesta- tions of the Zero-Point Energy (World Scientific, Singapore, 2001).

[6] E. M. Lifshitz, Zh. Eksp. Teor. Fiz. 29, 94 (1955) [Sov. Phys. JETP 2, 73 (1956)].

[7] T.H. Boyer, Phys. Rev. 174, 1764 (1968).

[8] L. L. DeRaad, Jr. and K. A. Milton, Ann. Phys. 186, 229 (1981)

[9] P. Gosdzinsky and A. Romeo, Phys. Lett. B 441, 265 
(1998)

[10] K. A. Milton, A. V. Nesterenko and V. V. Nesterenko, Phys. Rev. D 59, 105009 (1999)

[11] G. Lambiase, V. V. Nesterenko and M. Bordag, J. Math. Phys. 40, 6254 (1999)

[12] H. Razmi and N. Fadaei, Nucl. Phys. B 814, 582 (2009).

[13] I. Brevik and G. H. Nyland, Ann. Phys. 230, 321 (1994).

[14] V.V. Nesterenko and I.G. Pirozhenko, Phys. Rev. D 60, 125007 (1999).

[15] I. Cavero-Peláez and K. A. Milton, Ann. Phys. 320, 108 (2005); J. Phys. A 39, 6225 (2006)

[16] A. Romeo and K. A. Milton, Phys. Lett. B 621, 309 (2005); J. Phys. A 39, 6703 (2006)

[17] I. Cavero-Peláez, K. A. Milton and K. Kirsten, J. Phys. A 40, 3607 (2007)

[18] I. Brevik and A. Romeo, Phys. Scripta 76, 48 (2007)

[19] R. Balian and B. Duplantier, Ann. Phys. 112, 165 (1978).

[20] M. Bordag, V. Nesterenko, and I. Pirozhenko, Nucl. Phys. B 104 (2002).

[21] M. Bordag, V. V. Nesterenko, and I. G. Pirozhenko, Phys. Rev. D 65, 045011 (2002).

[22] J. S. Dowker and G. Kennedy, J. Phys. A 11, 895 (1978).

[23] D. Deutsch and P. Candelas, Phys. Rev. D 20, 3063 (1979).

[24] I. Brevik and M. Lygren, Ann. Phys. 251, 157 (1996).

[25] I. Brevik, M. Lygren and V. Marachevsky, Ann. Phys. 267, 134 (1998).

[26] I. Brevik, K. Pettersen, Ann. Phys. 291, 267 (2001).

[27] V. V. Nesterenko, G. Lambiase and G. Scarpetta, Ann. Phys. 298, 403 (2002).

[28] H. Razmi and S. M. Modarresi, Int. J. Theor. Phys. 44, 229 (2005).

[29] V. V. Nesterenko, G. Lambiase and G. Scarpetta, J. Math. Phys. 42, 1974 (2001).
[30] V. V. Nesterenko, I. G. Pirozhenko and J. Dittrich, Class. Quantum Grav. 20, 431 (2003).

[31] A. H. Rezaeian and A. A. Saharian, Class. Quant. Grav. 19, 3625 (2002).

[32] A. A. Saharian, Eur. Phys. J. C 52, 721 (2007).

[33] A. A. Saharian, in The Casimir Effect and Cosmology: A volume in honour of Professor Iver H. Brevik on the occasion of his 70th birthday, S. Odintsov et al. (eds.) (Tomsk State Pedagogical University Press, 2008), p.87, arXiv:0810.5207

[34] I. Brevik, S. Å. Ellingsen, and K. A. Milton, Phys. Rev. E 79, 041120 (2009).

[35] S. Å. Ellingsen, I. Brevik, and K. A. Milton, Phys. Rev. E 80, 021125 (2009).

[36] K. A. Milton, J. Wagner and K. Kirsten, Phys. Rev. D 80, 125028 (2009).

[37] J. Wagner, K. A. Milton and K. Kirsten, arXiv:0912.2374 [hep-th], to appear in the Proceedings of the 9th Conference on Quantum Field Theory Under the Influence of External Conditions (QFEXT09).

[38] I. Brevik, S. Å. Ellingsen, and K. A. Milton, arXiv:0911.2688 [hep-th], to appear in the Proceedings of the 9th Conference on Quantum Field Theory Under the Influence of External Conditions (QFEXT09).

[39] M. Abramowitz and I. A. Stegun Handbook of Mathematical Functions (New York: Dover, 1964).

[40] E. Elizalde, Ten Physical Applications of Spectral Zeta Functions (Berlin: Springer, 1995).

[41] C. M. Bender and S. A. Orszag, Advanced Mathematical Methods for Scientists and Engineers (Berlin: Springer, 1999) Chapter 8.2.

[42] S. A. Ellingsen, I. Brevik, J. S. Høye and K. A. Milton, Phys. Rev. E 78, 021117 (2008). 